\title{
Oncocytic lipoadenoma of parotid gland with sebaceous differentiation
}

\author{
Vadlamudi Swathi ${ }^{1, *}$, HR. Chandrasekhar ${ }^{2}$ \\ ${ }^{1}$ Senior Resident, Dept. of Pathology, GMC, Guntur, Andhra Pradesh, ${ }^{2}$ Professor, JJM Medical College, Davanagere, Karnataka, \\ India
}

*Corresponding Author:

Email: vadlamudi.swathi@yahoo.com

Received: $10^{\text {th }}$ October, 2017

Accepted: $14^{\text {th }}$ May, 2018

\begin{abstract}
Oncocytic lipoadenoma a rare benign neoplasm of salivary gland was first described by Hirokawa et al in submandibular gland. We report a case of oncocytic lipoadenoma (oncocytic sialolipoma) in a 69 year old male who presented with an asymptomatic mass in right parotid region for the past 3 months. Grossly the lesion was well encapsulated with homogenous mahogany brown with focal grey yellow areas. Microscopically the lesion was completely encapsulated and showed an intimate admixture of oncocytes and mature adipocytes in almost equal proportion, with areas of focal sebaceous differentiation.
\end{abstract}

Keywords: Salivary gland, Oncocytes, Oncocytic lipoadenoma.

\section{Introduction}

Oncocytic neoplasms of the salivary glands characterised histologically by a predominance of oncocytes consist of a wide array of morphologically diverse tumours. Salivary gland tumors with prominent oncocytic features are uncommon which includes oncocytoma, oncocytic carcinoma, and oncocytic cystadenoma, as well as oncocytic variants of pleomorphic adenoma, myoepithelioma, mucoepidermoid carcinoma, and epithelialmyoepithelial carcinoma. ${ }^{1}$

Oncocytic lipoadenomas of salivary gland with very few cases reported in past is an uncommon benign tumour which was not included in the 2005 WHO classification of tumours of salivary glands. In 1998 Hirokawa et al first described and proposed the term oncocytic lipoadenoma for this tumour in the submandibular gland. ${ }^{2}$ In this paper we describe an additional case of oncocytic lipoadenoma of parotid gland with sebaceous differentiation.

\section{Case Report}

A 69 year old male presented with three months history of an asymptomatic mass in the right parotid region. Though asymptomatic in view of short duration, size of the lesion and with a clinical suspicion of pleomorphic adenoma surgical resection was done.

\section{Histopathology}

Grossly the lesion was well-defined measuring $6 \times 3 \times 2 \mathrm{~cm}$ with a thin capsule. On cut section parenchyma of the mass was homogenous, mahogany brown with focal grey yellow areas. Normal salivary gland tissue was not made out grossly. Microscopically the tumour was well encapsulated composed of oncocytic cells arranged in lobulated acini. Oncocytes were round to polygonal in shape with abundant granular eosinophilic cytoplasm. Oncocytes were seen admixed with an almost equal proportion of mature adipocytes. Focal sebaceous differentiation noted. Chronic inflammatory infiltrate was seen. No evidence of atypia, necrosis or mitotic activity in the sections studied. The normal salivary gland was not seen. PTAH stain showed diffuse cytoplasmic granularity.

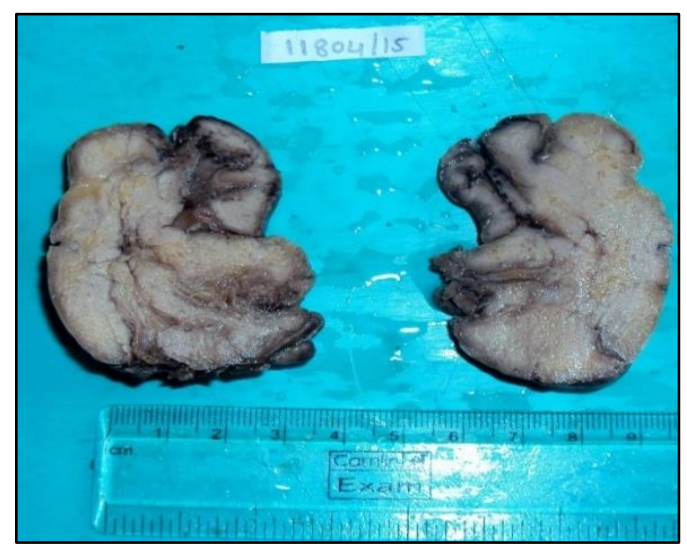

Fig. 1: Grossly oncocytic lipoadenoma with light brown cut surface and focal grey yellow areas

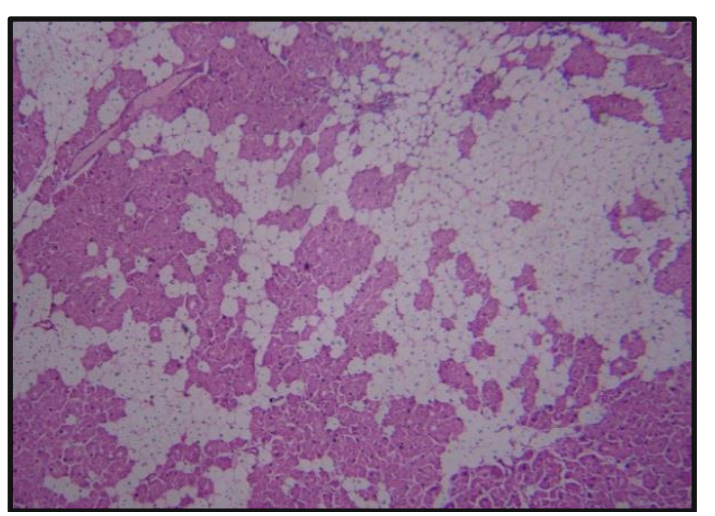

Fig. 2: Photomicrograph showing oncocytes admixed with mature adipocytes (H\&E x100) 


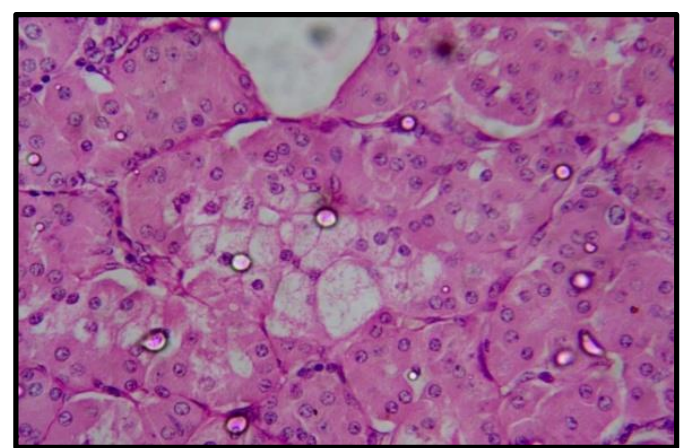

Fig. 3: Photomicrograph showing focal sebaceous differentiation (H\&E x400)

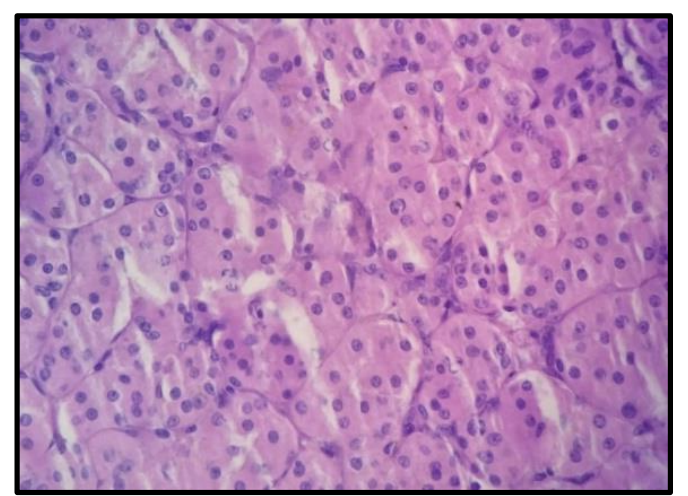

Fig. 4: Photomicrograph showing oncocytes arranged in acinar pattern with polygonal cells with abundant eosinophilic cytoplasm (H\&E x400)

\section{Discussion}

Oncocytes represent one of the special components of these neoplasms with their particular morphological features associated with increased mitochondrial content. Despite the quite common finding of oncocytic metaplasia of patients aged $\geq 70$ years, oncocytic tumours are rare. Oncocytic change is frequently observed in both non-neoplastic and neoplastic conditions of salivary glands varying from focal metaplasia of ducts and acini to oncocytosis and malignant lesions. $^{3}$

Lipoadenoma and adenolipoma described in many anatomic sites, including the skin, breast, parathyroid, thyroid, and salivary gland are designations used to refer to neoplasms composed of epithelial and lipomatous elements. ${ }^{1}$

Oncocytic lipoadenoma a recently described benign salivary gland neoplasm composed of oncocytic epithelium intimately admixed with mature adipose tissue. ${ }^{4}$ The World Health Organization Classification defines three categories of oncocytic neoplasms of the salivary glands: oncocytoma, nodular oncocytic hyperplasia (or oncocytosis), and oncocytic carcinoma. ${ }^{5}$

Lipomatous tumours admixed with salivary gland tissue attracted interest in recent years and include neoplastic and tumour-like lesions. Benign lipomatous tumours of the salivary glands with epithelial components include: Sialolipoma, lipoadenoma, oncocytic lipoadenoma, oncocytic lipoadenoma with sebaceous differentiation. ${ }^{5}$

Adipose tissue component in these tumours may be observed as a part of ageing, accompanying acinar atrophy and in lipomatosis. Adipose tissue may be present in primary tumours, such as sialolipoma, or as a component of benign tumours such as pleomorphic adenoma or myopeithelioma. Similar to oncocytic metaplasia, fatty change could be the expression of a metaplastic change of myoepithelial cells. ${ }^{3}$

Sebaceous differentiation in salivary glands other than sebaceous adenoma and sebaceous lymphadenoma, has been variously noted in pleomorphic adenoma, oncocytoma, silaloblastoma and warthin tumour. According to Klieb $\mathrm{HB}$ et al, oncocytic lipoadenoma to be added to the list of benign salivary gland neoplasms with the potential of developing sebaceous metaplasia. ${ }^{4}$

In our case amount of fatty tissue was about $50 \%$, admixed with oncocytes which showed diffuse cytoplasmic granular positivity with PTAH stain due to an abundance of mitochondria. Focal sebaceous differentiation noted. Nagao et $\mathrm{al}^{6}$ in a case series of seven cases of sialolipoma reported focal oncocytic metaplasia in a single case in parotid gland.

\section{Conclusion}

In view of rarity and new introduction in WHO classification we describe a case of oncocytic lipoadenoma of salivary glands adding on to few cases reported in the past. Awareness of this entity is helpful during the evaluation of patients with salivary gland neoplasms.

\section{References}

1. Lau SK, Thompson LD. Oncocytic lipoadenoma of the salivary gland: a clinicopathologic analysis of 7 cases and review of the literature. Head Neck Pathol. 2015;9:39-46.

2. Hirokawa M, Shimizu M, Manabe T, Ito J, Ogawa S. Oncocytic lipoadenoma of the submandibular gland. Hum Pathol. 1998;29:410-2.

3. Casadei GP, Collina G, Salerno A, Canani FB, Dall'olio D, Lega S. Oncocytic lipoadenomatous tumours of salivary glands. Histopatholgy. 2012;61:1225-7.

4. Klieb HB, Perez-Ordon ez B. Oncocytic lipoadenoma of the parotid gland with sebaceous differentiation. Study of its keratin profile. Virchows Arch. 2006;449:722-5.

5. Pusiol T, Franceschetti I, Scialpi M, Piscioli I. Oncocytic sialolipoma of the submandibular gland with sebaceous differentiation: a new pathological entity. Indian J Pathol Microbiol. 2009;52:379-82.

6. Nagao T, Sugano I, Ishida Y, Asoh A, Munakata S, Yamazaki K, et al. Sialolipoma: A report of seven cases of a new variant of salivary gland lipoma. Histopathology. 2001;38:30-6.

How to cite this article: Swathi V, Chandrasekhar HR. Oncocytic lipoadenoma of parotid gland with sebaceous differentiation. Ind J Pathol Oncol, 2018;5(3):516-517. 